\title{
A NOTE ON UNIQUENESS OF L-FUNCTIONS WITH REGARD TO MULTIPLICITY
}

\author{
HARINA P. WAGHAMORE ${ }^{\dagger}$ AND VIJAYLAXMI S.B.
}

\author{
Date of Receiving : $\quad$ : 02.08 .2019 \\ Date of Revision : $\quad$ : 06.12 .2019 \\ Date of Acceptance : 13.12 .2019
}

\begin{abstract}
By introducing the concept of multiplicity along with weighted sharing of small functions or fixed points, we investigate the uniqueness problems of meromorphic function and a $L$-function in the extended Selberg class. The results obtained in this paper improves the results due to Liu-Li-Yi ([14]), Sahoo-Halder ([18]), F. Liu-Li-Yi ([15]) and Wen-Jie Hao and Jun-Fan Chen ([3]).
\end{abstract}

\section{Introduction and main results}

Selberg studied that the Riemann hypothesis is also true for $L$-functions in the Selberg class. An $L$-function based on Riemann Zeta function as the prototype is defined as Dirichlet series

$$
L(s)=\sum_{n=1}^{\infty} a(n) n^{-s}
$$

of a complex number $s=\sigma+i t$ satisfying the following axioms([19]):

(i) Ramanujan hypothesis: $a(n) \ll n^{\epsilon}$ for every $\epsilon>0$;

(ii) Analytic continuation: There is a non-negative integer $m$ such that $(s-1)^{m} L(s)$ is an entire function of finite order;

(iii) Functional equation: $L$ satisfies a functional equation of type

$$
\Lambda_{L}(s)=\omega \overline{\Lambda_{L}(1-\bar{s})},
$$

where

$$
\Lambda_{L}(s)=L(s) Q^{s} \prod_{j=1}^{k} \Gamma\left(\lambda_{j} s+\nu_{j}\right)
$$

with positive real numbers $Q, \lambda_{j}$ and complex numbers $\nu_{j}, \omega$ with $R e \nu_{j} \geq 0$ and $|\omega|=1$;

2010 Mathematics Subject Classification. 30D35.

Key words and phrases. L-functions, Weighted sharing, Multiplicity.

Communicated by: Nikhil Khanna

${ }^{\dagger}$ Corresponding author. 